\title{
Numerical Modeling of the Configuration of a Long-distance Free-spanning Submarine Pipeline on an Uneven Seabed
}

\author{
Leige $\mathrm{Xu}$ \\ North China University of Water Resources and Electric Power \\ Zhengzhou, China \\ Mian Lin \\ Institute of Mechanics, Chinese Academy of Sciences \\ Beijing, China
}

\begin{abstract}
In this paper, a procedure is developed to analyze long-distance free-spanning pipelines on an uneven seabed through the incorporation of the Vector Form Intrinsic Finite Element (VFIFE or V-5) method with the bubble model, which is also called the UWAPIPE model. A dynamic contacting scheme is proposed to model variations of the contact situation between a seabed and a deforming pipeline on it. Specifically, a high-efficiency Message Passing Interface (MPI) parallel scheme is adopted to reduce the computing time of the procedure. Through the use of the procedure, the configuration of a $10 \mathrm{~km}$ long-distance pipeline lying on a real irregular seabed in the South China Sea is simulated, and the results are compared with a Remotely Operated Underwater Vehicle (ROV) survey and Det Norske Veritas (DNV) recommendations. The effects of the submerged weight and internal pressure of the pipeline are evaluated. Subsequently, the unevenness ratio is defined, and its relation to the pipeline bending moment is presented. The effects of the seabed undulation and adjacent spans are proved to be significant for the examined case.
\end{abstract}

\section{INTRODUCTION}

Submarine pipelines commonly cross uneven seabed, and free spans could form due to the seabed undulations in practical engineering. In the conventional security assessment for free-spanning pipelines, only the span height and length are considered, while the effects of the seabed configuration and the resulting bending stresses are ignored (Rezazadeh et al., 2010). This would produce errors in the analysis of vortex-induced vibration, global buckling, axial walking, and even the integrity of the pipeline. To consider the effect of the uneven seabed, the pipeline configuration of the static equilibrium, including the free span scenarios, penetration in the soil, and corresponding stress distribution under different work conditions (e.g., as-laid, water-filled, and operational) must be obtained first (DNV, 2006; Larsen et al., 2002; Soreide et al., 2005).

The pipe-soil interaction plays a significant role in the evaluation of the global pipeline stability. In many previous studies, a series of nonlinear springs are used to model the soil resistances in the axial, lateral, and vertical directions, respectively (Gao et al., 2011; Larsen et al., 2002; Pereira et al., 2008; Sun et al., 2009; Wang et al., 2015). While the simplicity of the spring model is acknowledged, it is difficult to predict the actual pipeline embedment and the corresponding vertical soil resistance through the use of the idealized springs because they cannot reflect the actual soil deformation, especially the plastic component. Moreover, the spring model commonly relates the lateral and axial resistances to the pipeline submerged weight instead of to the varying vertical soil resistance (Tian and Cassidy, 2008). To account for these effects, Tian and Cassidy $(2008,2009,2010)$ proposed and developed a series of force-resultant pipe-soil interaction models, one of which, the UWAPIPE model or the so-called bubble model, was integrated with the Finite Element Method (FEM) platform

Received April 11, 2016; revised manuscript received by the editors August 9, 2016. The original version was submitted directly to the Journal.

KEY WORDS: VFIFE method, pipe-soil interaction, free-spanning pipeline, uneven seabed.
ABAQUS to simulate exposed pipelines on sandy seabed. In these works, variations of the pipeline embedment and the corresponding mobilized soil resistance along the pipeline are remarkable. Besides, the contacting situation between the pipeline and the seabed is predetermined and cannot change during the simulation. Nevertheless, for a pipeline on an uneven seabed, the contacting situation changes with the deforming pipeline, and this can influence the pipeline response. Therefore, a dynamic contacting scheme is adopted in this paper to update the contacting situation between the deforming pipeline and the seabed.

The FEM was used by many researchers to obtain the static equilibrium pipeline configuration (Chung and Cheng, 1996; Chung et al., 1995; Fan, 2013; Larsen et al., 2002; Pereira et al., 2008; Soreide et al., 2005). To deal with the geometric nonlinearity of the slender pipeline, the nonlinear matrix and substep iterations are always necessary for the conventional FEM. For contacting nonlinear problems, Lagrangian techniques are commonly introduced into the conventional FEM (Ma et al., 2014). Although it is applicable and practicable, this algorithm is complicated, the simplicity of the FEM is lost, and particularly, the programming work becomes tedious.

In recent years, Ting et al. (Shih et al., 2004; Ting et al., 2004a, 2004b) proposed a Vector Form Intrinsic Finite Element (VFIFE or V-5) method. A VFIFE 3D beam element presented by Ting et al. (2012) is used in this paper, and it can model the axial deformation, biaxial bending, and torsion of the pipeline. For the simulation of long-distance pipelines, the VFIFE method has several advantages. Substep iterations are not required in nonlinear structural analysis due to the inherited predictor-corrector mechanism in the VFIFE method (Wang et al., 2006). The displacement of an element is composed of rigid motion and the deformation of the element (Lien et al., 2010). In the VFIFE method, the rigid element motion is eliminated by the fictitious reversed movement, and the element inner forces are directly calculated by the element deformation. Because of the use of the fictitious reversed movement, the procedure for problems with geometric nonlinearity or rigid-body motion is the same as that for linear problems with the VFIFE method. Moreover, the VFIFE method combines particle dynamics and the FEM. The nodes' motion is governed 